\title{
Enhanced method for the reconstruction of zero-dispersion wavelength maps of optical fibers by measurement of continuous-wave four-wave mixing efficiency
}

\author{
Miguel González-Herráez, Pedro Corredera, Maria L. Hernanz, and Jose A. Méndez
}

\begin{abstract}
The zero-dispersion wavelength map of an optical fiber can be obtained from measurement of end-to-end four-wave mixing efficiency at various wavelengths. A fast and unambiguous algorithm for reconstruction of the zero-dispersion wavelength map of an optical fiber by measurement of four-wave mixing efficiency is proposed. This method can produce high-resolution results in a few seconds. We also study the limitations of this technique that are due to polarization-mode dispersion (PMD). Simple practical rules to avoid the effects of PMD in such measurements are established. (c) 2002 Optical Society of America

OCIS codes: $\quad 060.4370,000.3860,060.2270,190.4380,190.4410$.
\end{abstract}

\section{Introduction}

With increasing power guided by fiber-optic-based communication systems, a large number of techniques to avoid nonlinearities in optical fibers have emerged during the past few years. Among these, dispersion management has become an important tool to provide low overall dispersion while preventing transmission in the zero-dispersion wavelength region of the fiber where four-wave mixing causes impairment in communication systems because of cross talk. For this purpose, precise knowledge of the mean zero-dispersion wavelength of the fiber is not enough, and knowledge of the variation of this parameter along the fiber is required.

In previous publications several techniques were reported that are capable of producing the zerodispersion wavelength map of an optical fiber. ${ }^{1-4}$ Mollenauer et $a .^{2}$ constructed an optical timedomain reflectometer-like apparatus that is capable of making distributed measurements of chromatic

The authors are with the Instituto de Física Aplicada, Consejo Superior de Investigaciones Cientificas, Serrano 144, 28006 Madrid, Spain. M. González-Herráez's e-mail address is ltqg340@ cetef.csic.es.

Received 8 January 2002; revised manuscript received 12 March 2002.

0003-6935/02/193796-08\$15.00/0

(C) 2002 Optical Society of America dispersion with an accuracy of $\pm 0.03 \mathrm{ps} \mathrm{nm}^{-1} \mathrm{~km}^{-1}$ ( $\pm 0.5 \mathrm{~nm}$ in $\lambda_{0}$ for a typical dispersion-shifted fiber) and a spatial resolution of $500 \mathrm{~m}$. Eiselt et al. ${ }^{4} \mathrm{dem}-$ onstrated a method for zero-dispersion wavelength mapping in optical fibers by using the generation of a four-wave mixing product through a pulse-overlap technique. They reported a spatial resolution of $700 \mathrm{~m}$, and they pointed out that a spatial resolution of $70 \mathrm{~m}$ would be feasible although it would probably require a substantial modification of the pulsegeneration scheme. Brener et $a l .{ }^{3}$ demonstrated a method for extraction of zero-dispersion wavelength variations along the fiber by measurement of continuous-wave four-wave mixing efficiency with two tunable lasers. This method can also be implemented with a single tunable laser source.5,6 The achievable results are similar to those described by Brener et al., ${ }^{3}$ but several problems arise with the reconstruction procedure. ${ }^{7}$ In general, the constant detuning scheme (two tunable lasers) should be preferred. Of all the methods described, this one has significant advantages: the measurement setup is simple, it can yield results with very high spatial resolution $(\simeq 100 \mathrm{~m})$, and it is valid for any range of values of $\lambda_{0}$. However, the fitting procedure is time consuming and the results obtained can be ambiguous. In this paper we overcome these difficulties by proposing a new algorithm for reconstruction of the zero-dispersion wavelength profiles of optical fibers by measurement of four-wave mixing efficiency at 
several wavelengths. We show that this method is applicable to both normal-dispersion and dispersionshifted fibers.

\section{Overview of the Problem}

Four-wave mixing: (FWM) is generated when three optical carriers at different frequencies $\left(f_{i}, f_{j}, f_{k}\right)$ interact through the third-order electric susceptibility of the optical fiber. A fourth carrier is thus generated whose frequency is a linear combination of the other three $\left(f_{w}=f_{i}+f_{j}-f_{k}\right)$. A particular case arises when only two frequencies interact through the fiber. The equations that govern this so-called degenerate case are the same as those that describe the more general case but for $f_{i}=f_{j}$. The new frequency appears, therefore, at $f_{w}=2 f_{i}-f_{k}$. All the subsequent discussion refers to this case.

The power of four-wave mixing is given by the product of three terms:

$$
\begin{aligned}
P_{w}= & F_{P}\left(\gamma, \alpha, P_{i}, P_{k}, L\right) \eta\left(D_{c}, \Delta \lambda, \alpha, P_{i}, P_{k}, L\right) \\
& \times T_{\mathrm{pol}}\left(\mathbf{s}_{i}, \mathbf{s}_{k}\right) .
\end{aligned}
$$

The first term $\left(F_{P}\right)$ is a power term that depends on the nonlinear characteristics of the fiber $(\gamma)$, the attenuation $(\alpha)$, the length of the fiber $(L)$, and the input powers of the two wavelengths $\left(P_{i}\right.$ and $\left.P_{k}\right)$. The second term $(\eta)$ is a phase-matching efficiency term. For reasonably low powers ${ }^{9}$ it can be considered to depend only on the phase mismatch among the three waves involved:

$$
\eta=\frac{\alpha^{2}}{\alpha^{2}+(\Delta \beta)^{2}}\left\{1+\frac{4 \exp (-\alpha L) \sin ^{2}(\Delta \beta L / 2)}{[1-\exp (-\alpha L)]^{2}}\right\},
$$

where $\Delta \beta$ is the phase mismatch. This phase mismatch depends strongly on the chromatic dispersion characteristics of the fiber $\left(D_{c}\right)$. In the vicinity of the zero-dispersion wavelength and neglecting fiber nonuniformities and second- and higher-order derivatives of dispersion, we can write the phase-mismatch term as

$$
\begin{aligned}
\Delta \beta & =\beta\left(\lambda_{w}\right)+\beta\left(\lambda_{k}\right)-2 \beta\left(\lambda_{i}\right) \\
& =-\frac{2 \pi c}{\lambda^{2}} \frac{\mathrm{d} D_{c}}{\mathrm{~d} \lambda} 2\left(\lambda_{i}-\lambda_{k}\right)^{2}\left(\lambda_{i}-\lambda_{0}\right) .
\end{aligned}
$$

Efficiency $\eta$ as it is expressed in Eq. (2) is maximum when the phase-matching condition is achieved, that is, when $\Delta \beta$ is zero.

The third term in Eq. (1) is a polarization transfer function term, and it can be written as

$$
T_{\mathrm{pol}}\left(\mathbf{s}_{i}, \mathbf{s}_{k}\right)=1 / 2\left(1+\mathbf{s}_{i} \cdot \mathbf{s}_{k}\right),
$$

where $\mathbf{s}_{i}$ and $\mathbf{s}_{k}$ are the polarization states of the incident waves in the Poincare sphere. This polarization term is unity when the polarization states of the two waves are perfectly parallel $\left(\mathbf{s}_{i}=\mathbf{s}_{k}\right)$.

The previous model for phase-matching efficiency is not rigorous when one considers random fluctua- tions in the zero-dispersion wavelength along the fiber. Previous measurements already demonstrated that the zero-dispersion wavelength varies along the fiber in an unpredictable manner. ${ }^{10}$ Karlsson $^{11}$ has proposed a statistical description of the evolution of zero-dispersion wavelength along an optical fiber. In this model the zero-dispersion wavelength is treated as the sum of a constant zero-dispersion and strictly stationary stochastic process with zero mean and a Gaussian probability-density function:

$$
\lambda_{0}(z)=\lambda_{0}{ }^{\text {const }}+\lambda_{0}{ }^{\text {rand }}(z) .
$$

As with any other stochastic process, its correlation length may be defined:

$$
L_{c}=\int_{0}^{\infty} R_{\lambda}(\zeta) \frac{\mathrm{d} \zeta}{R_{\lambda}(0)},
$$

where $R_{\lambda}(\zeta)$ is the autocorrelation of $\lambda_{0}{ }^{\operatorname{rand}}(z) . \quad L_{c}$ measures the rate of change of the stochastic process; a small value of $L_{c}$ means quick changes in the zerodispersion wavelength profile, and a large $L_{c}$ means slow changes. Based on this variable, Karlsson divides the perturbations that affect the fiber into two types: short-length variations (which are due to small, local changes in the drawing process and should have correlation lengths of $L_{c} \sim 1 \mathrm{~m}$ and standard deviations of $\sigma \sim 6 \mathrm{~nm}$ ) and long-length variations that are due to slow drifts in the fabrication process $\left(L_{c} \sim 1 \mathrm{~km} ; \sigma \sim 2 \mathrm{~nm}\right)$. Our preliminary measurements with the single tunable laser scheme ${ }^{7}$ are in agreement with these values. Short-term fluctuations have little effect on end-to-end FWM efficiency because $\left.\left.\Delta \beta L_{c}\right|_{\text {long-term }} \gg \Delta \beta L_{c}\right|_{\text {short-term }}$. From here on, our model of nonuniform fiber will consider only long-term fluctuations $\left(L_{c} \geq 500 \mathrm{~m}\right)$.

Assuming constant input powers and negligible polarization-mode dispersion (PMD), the spectral dependence of the FWM effect is given directly by phase-matching efficiency and, more specifically, by chromatic dispersion.

For a nonuniform fiber, however, the expression of the phase-matching efficiency term depends on the distribution of the dispersion parameters along the fiber. If we further assume that the dispersion is a first-order approximation and that the dispersion slope along the fiber is constant, the expression for the intensity of the FWM product at the output of the fiber can be written $a^{3}$

$$
I_{\mathrm{FWM}}\left(\lambda_{1}\right) \propto\left|\int_{0}^{L} \exp [i \phi(z)] \exp \left(-i \kappa \lambda_{1} z\right) \exp (-\alpha z) \mathrm{d} z\right|^{2},
$$

where $\phi(z)$ and $\kappa$ are

$$
\phi(z)=\kappa \int_{0}^{z} \lambda_{0}(y) \mathrm{d} y, \quad \kappa=2 \pi c\left(\frac{\Delta \lambda}{\lambda}\right)^{2} \frac{\mathrm{d} D}{\mathrm{~d} \lambda},
$$




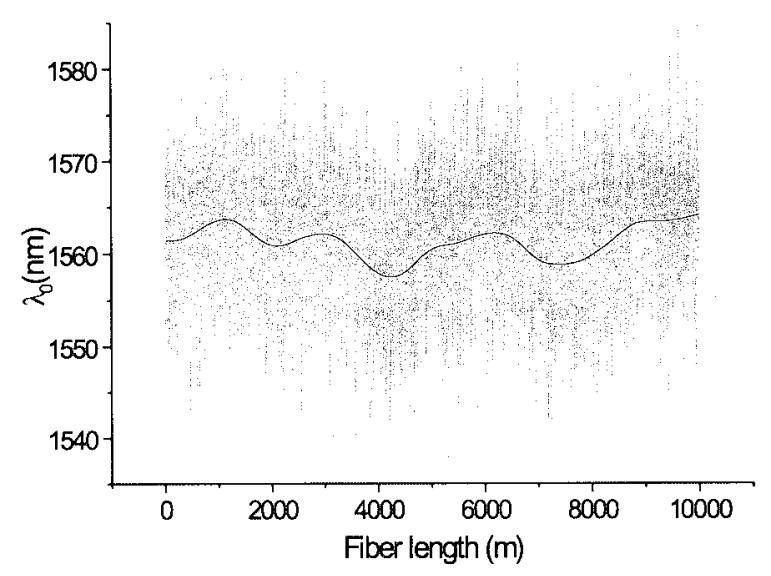

(a)

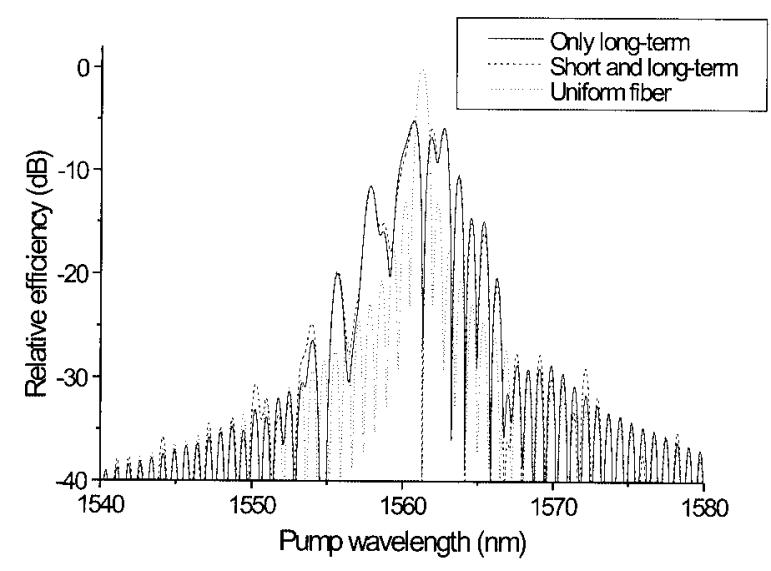

(b)

Fig. 1. (a) Zero-dispersion wavelength map of a nonuniform fiber, including and excluding short-term variations. (b) FWM efficiency curve $(\Delta \lambda=4 \mathrm{~nm})$ for the simulated fiber including and neglecting short-term fluctuations versus the efficiency curve for a fiber with constant zero-dispersion wavelength (dotted curve). The slight differences that appear between the two cases are due to small long-term perturbations induced by the simulated shortterm perturbations. Note the wavelength shift of the peak FWM efficiency and the decrease of $\sim 5 \mathrm{~dB}$ in the peak gain.

where $\Delta \lambda$ is the difference between the two wavelengths injected into the fiber. When $\kappa$ is constant, (that is, with constant $\Delta \lambda$ between the two wavelengths at the input), expression (7) becomes the squared modulus of a Fourier transform, in which $\exp \left(-i \kappa \lambda_{1} z\right)$ is the nucleus of the transform. Note that the suppositions stated above can be considered realistic, at least in the wavelength range in which the FWM efficiency is not zero. For instance, the error in chromatic dispersion that is due to neglecting second-order dispersion in a $30-\mathrm{nm}$ band about the zero-dispersion wavelength is less than $1 \%$. The error that is due to assuming a constant dispersion slope along the fiber has been proved to be less than $2 \%$. $^{3}$

Figure 1 shows the effect of random variations of $\lambda_{0}$ on FWM efficiency. The simulated zero-dispersion wavelength profile has been generated with the ad- dition of long-term variations with $L_{c} \sim 1.5 \mathrm{~km}$ and short-term fluctuations of $L_{c} \sim 1 \mathrm{~m}$. For wavelength detunings as big as $10 \mathrm{~nm}$, short-term variations do not induce significant changes in the phase mismatch, and only long-term variations have visible effects on FWM efficiency. In a nonuniform fiber, in general, there is a wavelength shift and a loss in peak efficiency, and the shape of the efficiency curve broadens by approximately the same amount as the variations in the zero-dispersion wavelength.

Our problem of interest can be stated in this way: Given the intensity of the four-wave mixing product at different wavelengths $\left[I_{\mathrm{FWM}}\left(\lambda_{1}\right)\right]$, find the zerodispersion wavelength map of the optical fiber under study. Following Brener's notation, the problem can be rewritten in simplified notation:

$$
I_{\mathrm{FWM}}(q) \propto\left|\int_{0}^{L} g(z) \exp (-i q z) \mathrm{d} z\right|^{2},
$$

where $g(z)$ is a complex function whose modulus for each $z$ is proportional to $\exp (-\alpha z)$. We have replaced $\kappa \lambda_{1}$ with $q$, whose value is given in units of a wave vector. The problem of interest is quite similar to the classic problem of phase retrieval: Retrieve an object $g(z)$ from the magnitude of its Fourier transform. This problem has been treated extensively in the past 20 years. ${ }^{12,13}$ However, with respect to the classic problem, ours has two significant advantages: First, this problem is known to have a unique solution ${ }^{3}$; second, attenuation $\alpha$ can easily be known for all $z$.

\section{Development of the New Algorithm}

One of the most successful algorithms used for the phase-retrieval problem has been Fienup's errorreduction algorithm and a later modification of it known as the input-output algorithm. ${ }^{12}$ The errorreduction algorithm is iterative, and it can be summarized as follows: In the $k$ th iteration an initial estimate of the object $\left[g_{k}(z)\right]$ is Fourier transformed, yielding the transform $G_{k}(q)$. The phase of the resultant transform $\left[\Phi_{k}(q)\right]$ is used as an estimation of the phase of the Fourier transform of the real object, so a new, estimated spectrum $\left\{\sqrt{I(q)} \exp \left[j \Phi_{k}(q)\right]\right\}$ can be inversely transformed to yield a new estimation of the object $\left[g_{k}{ }^{\prime}(z)\right]$. For the $(k+1)$ th iteration the initial estimate $\left[g_{k+1}(z)\right]$ will be obtained from the output of the previous iteration by use of the following rule ${ }^{12}$ :

$$
g_{k+1}(z)=\left\{\begin{array}{ll}
g_{k}{ }^{\prime}(z) & z \notin \gamma \\
0 & z \in \gamma
\end{array},\right.
$$

where $\gamma$ includes all the points that violate the constraints imposed on the object as a result of our previous knowledge of its properties. The points that violate the constraints include those that result from the object's size (support constraints) and also those for which $g_{k-1}{ }^{\prime}(z)$ does not satisfy the mathematical restrictions imposed on $g(z)$. It can be shown that the mean-squared error between $\left|G_{k}(q)\right|$ and $\sqrt{I(q)}$ 
decreases in each iteration and that, therefore, if a sufficiently large number of iterations is performed, the error will always converge to the minimum-error solution. The input-output approach is similar to the error-reduction approach, but here a new rule is established to produce $g_{k+1}(z)$ from $g_{k}{ }^{\prime}(z)$ :

$$
g_{k+1}(z)= \begin{cases}g_{k}{ }^{\prime}(z) & z \notin \gamma \\ g_{k}(z)-\beta g_{k}{ }^{\prime}(z) & z \in \gamma\end{cases}
$$

where $\beta$ is a constant.

Following the same philosophy as for the last two algorithms, we developed a new algorithm to overcome the problem associated with zero-dispersion wavelength mapping from FWM efficiency as was described in Section 2. From now on we shall call this algorithm the modified Fienup algorithm (MFA). The basic process remains the same, but we impose two new restrictions on function $g(z)$ : First, it has only nonzero values in the range $[0, L]$; second, the modulus at each $z$ is given by $\exp (-\alpha z)$. Note that $L$ and $\alpha$ can easily be obtained from optical timedomain reflectometer measurements. Therefore the new rule for the initial estimation of the $(k+1)$ th iteration is

$$
g_{k+1}(z)=\left\{\begin{array}{cl}
\exp (-\alpha z) \exp \left[j \phi_{k}{ }^{\prime}(z)\right] & z \in[0, L] \\
0 & z \notin[0, L]
\end{array}\right.
$$

where $\phi_{k}{ }^{\prime}(z)$ stands for the phase of $g_{k}{ }^{\prime}(z)$. We claim that this algorithm is fast and robust and that it yields precise and high-resolution results, as is shown in Section 4 below.

To clarify concepts, we have explicitly written the steps of our algorithm:

1. The starting data are a set of $N$ samples of the normalized FWM efficiency measured at a constant wavelength detuning (constant $\Delta \lambda$ ) in the range $\left[\lambda_{\min }, \lambda_{\max }\right]$. From this efficiency, form an equivalent system in terms of the variable $q$ (remember that $\left.q=\kappa \lambda_{1}\right)$. For simplicity, we work with a $q$-shifted (equivalently $\lambda$-shifted) version of the efficiency. We call the resulting efficiency $I(q)$. The idea is that the shift in $\lambda$ should be reasonably close to the mean zero-dispersion wavelength. In this case the resolved phase changes should be smaller and the speed of convergence should be higher. Note that in our algorithm we have to work with the square root of the efficiency $[\sqrt{I(q)}]$.

2. Form an initial estimate of the object $g_{0}(z)$. We find the easiest estimate by setting $g_{0}(z)=$ $\exp (-\alpha z)$.

3. Generate the Fourier transform of the previous estimate. For iteration $k$ of the algorithm we call this transform $G_{k}(q)$. The phase of this transform $\left[\Phi_{k}(q)\right]$ is used as an estimate of the true phase of the FWM product.

4. Construct a new Fourier pattern, $G_{k}{ }^{\prime}(q)=$ $\sqrt{I(q)} \exp \left[j \Phi_{k}(q)\right]$. Inverse Fourier transform the pattern to achieve a new object, $g_{k}{ }^{\prime}(z)$.
5. Generate the initial estimation for the next iteration, using the rule described in Eq. (12). Start again from step 3, or stop if the maximum number of iterations has been achieved.

The spatial resolution of this algorithm depends on the wavelength range $\left[\lambda_{\min }, \lambda_{\max }\right]$ of significant measurements. In fact, the spatial resolution in this case reads as $\Delta x=2 \pi / \kappa\left(\lambda_{\min }-\lambda_{\max }\right)$.

\section{Simulation Results}

Using these ideas, we developed a fast-Fouriertransform-based application for the reconstruction of an arbitrary zero-dispersion wavelength map. The performance of the MFA was compared to the errorminimization technique by use of a Gauss-Newton algorithm. In both cases the initial guess consisted of a fiber with constant $\lambda_{0}$. Consistent improvement in both the precision and the resolution of the results as well as in the speed of convergence was achieved. To illustrate the capacity of our algorithm we considered several zero-dispersion wavelength maps and their respective FWM efficiency curves. From the FWM efficiency curves we simulated the retrieval of the original zero-dispersion wavelength maps under three conditions: no noise and perfect knowledge of the fiber parameters, noise in the FWM efficiency curve with perfect knowledge of the fiber parameters, and noise in the FWM efficiency curve and imperfect knowledge of the fiber parameters. We performed our tests in normal-dispersion fibers and in dispersion-shifted fibers.

\section{A. Four-Wave Mixing Efficiency Curves with No Noise}

An example of the reconstruction algorithm is shown in Fig. 2 for a fiber with normal dispersion. The parameters used for this fiber were $\alpha=0.32 \mathrm{~dB} / \mathrm{km}$, $\mathrm{d} D_{c} / \mathrm{d} \lambda=0.09 \mathrm{ps} \mathrm{nm}^{-2} \mathrm{~km}^{-1}$, and $L=10 \mathrm{~km}$. The efficiency was generated by use of a wavelength detuning $(\Delta \lambda)$ of $3 \mathrm{~nm}$. The measured wavelength spanned $\lambda_{\text {min }}=1300$ to $\lambda_{\max }=1324 \mathrm{~nm}$. The mean zero-dispersion wavelength was $1311.2 \mathrm{~nm}$. Results after only 300 iterations ( $\sim 10 \mathrm{~s}$ of processing time with the fast-Fourier-transform-based algorithm) are shown. We compare these results with those obtained with a plain Gauss-Newton search algorithm, expanding the zero-dispersion wavelength profile into a set of orthogonal functions and minimizing the mean-squared error between the two efficiencies in a way similar to that of Brener et al. ${ }^{3}$ For large correlation lengths, both algorithms yield good results, although our algorithm requires fewer iterations for convergence. For smaller correlation lengths, however, the Gauss-Newton algorithm yields bad results (Fig. 3) because the resolution in this case is limited by the number of coefficients used in the fitting. A similar effect can be observed when $\Delta \lambda$ is increased. We tried to increase the number of coefficients used in the Gauss-Newton fitting, but the results then 


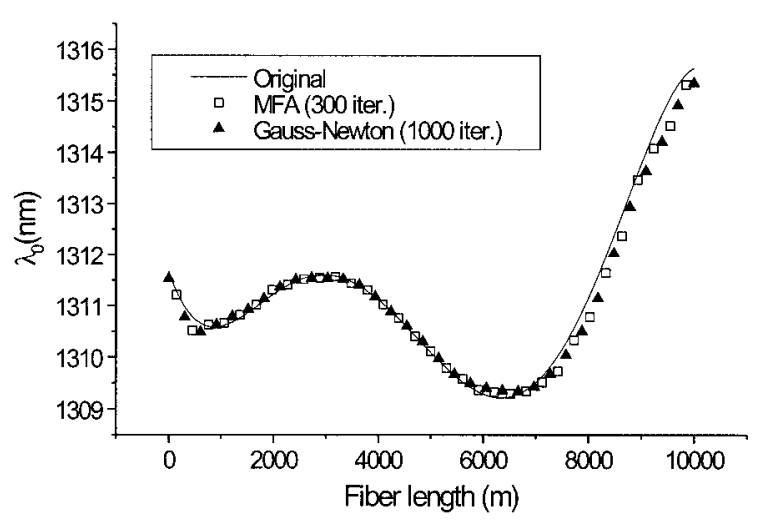

(a)

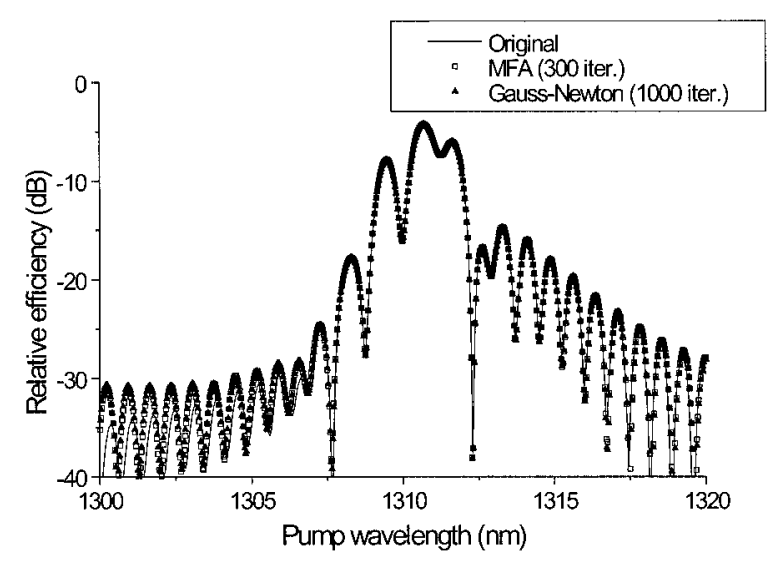

(b)

Fig. 2. (a) Zero-dispersion wavelength map of nonuniform fiber with $L_{c} \sim 3 \mathrm{~km}$ and profile reconstructed by the new algorithm and by the Gauss-Newton algorithm. (b) FWM efficiency curve for the simulated fiber and FWM efficiency of the fiber reconstructed by both methods.

became noisy and required a great number of iterations for convergence (as many as 3000 or more, involving processing times of more than $1 \mathrm{~min}$ ). For both algorithms, however, with very large $\Delta \lambda s$ ( $\geq 8 \mathrm{~nm})$ an impractically large number of iterations is required for convergence. It is interesting to say that the algorithm can be used for fibers with discontinuities (for example, several fibers spliced together). In fact, this algorithm can cope better with discontinuous profiles than can the error-minimization approach because the resolution in this case is not limited to the number of coefficients used in the fitting.

Similar tests were performed on dispersion-shifted fibers (DSFs). We obtained results similar to those obtained for normal-dispersion fibers. An example of a reconstruction is shown in Fig. 4. The parameters used for this fiber were $\alpha=0.187 \mathrm{~dB} / \mathrm{km}$, $\mathrm{d} D_{c} / \mathrm{d} \lambda=0.067 \mathrm{ps} \mathrm{nm}^{-2} \mathrm{~km}^{-1}$, and $L=10 \mathrm{~km}$. The efficiency was generated with a wavelength detuning $(\Delta \lambda)$ of $4 \mathrm{~nm}$. The measured wavelength span was $\lambda_{\min }=1550$ to $\lambda_{\max }=1574 \mathrm{~nm}$. The mean zerodispersion wavelength was $1561.2 \mathrm{~nm}$.

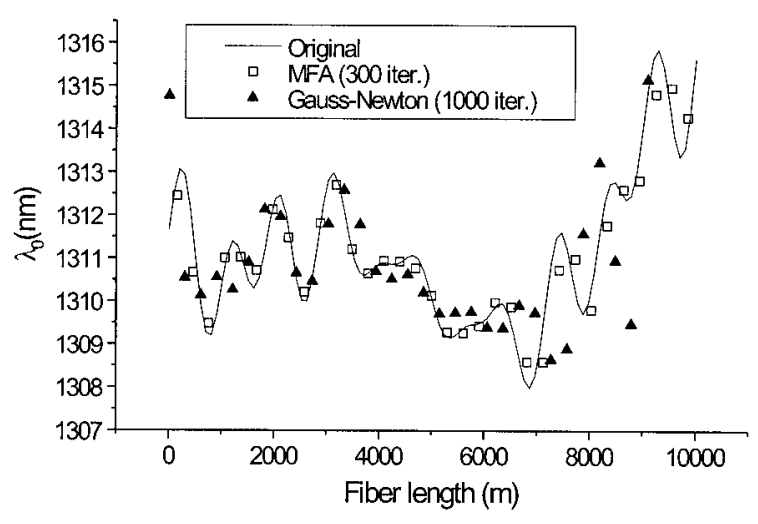

(a)

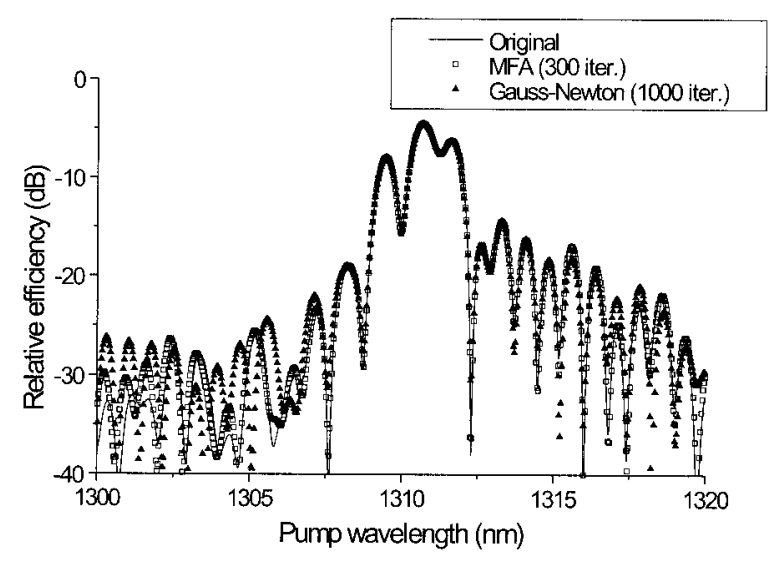

(b)

Fig. 3. (a) Zero-dispersion wavelength map of nonuniform fiber with $L_{c} \sim 1 \mathrm{~km}$ and profile reconstructed by the new algorithm and by the Gauss-Newton algorithm. (b) FWM efficiency curve for the simulated fiber and FWM efficiency of the fiber reconstructed by both methods.

\section{B. Four-Wave Mixing Efficiency Curves with Measurement Noise}

To check the robustness of this algorithm to noise, we simulated detection noise in the measurements. We assumed a Gaussian perturbation in the detected current caused by thermal noise (with constant $\sigma$ for all the wavelengths). The measured power was assumed to be proportional to the rms value of the detected photocurrent. This noise restriction was equivalent to considering a dynamic range limitation of approximately $10 \log \left(I_{\max } / \sigma\right)$. We made our tests considering the same DSF as we considered in the last example of Subsection 4.A. The algorithm showed an impressive capacity to overcome noise limitations. Figure 5 shows a sample reconstruction with the same DSF fiber that was used in the last example of Subsection 4.A. In this case a maximum acceptable noise of $\sigma / I_{\max }=0.01$ [dynamic range (D.R.), $20 \mathrm{~dB}$ ] was achieved. We note that for this example the rms power of the simulated noise perturbation was bigger than the power recorded for more than $50 \%$ of the points. 


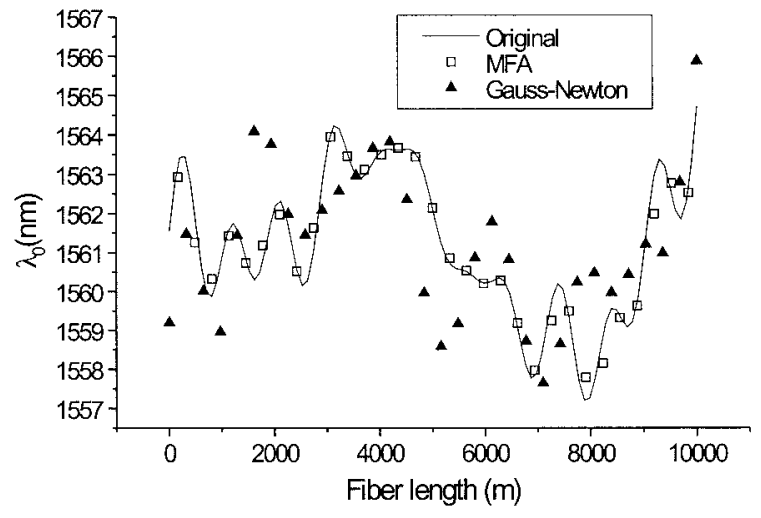

(a)

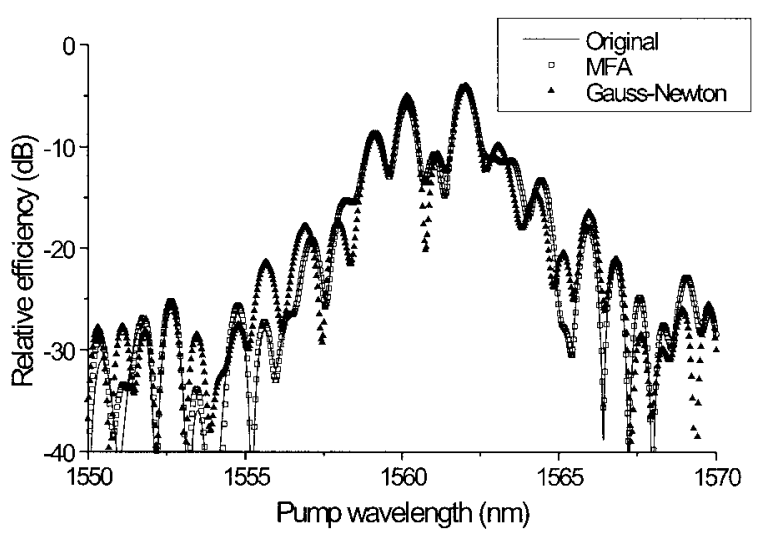

(b)

Fig. 4. (a) Zero-dispersion wavelength map of nonuniform fiber with $L_{c} \sim 1 \mathrm{~km}$ in the 1550-nm window and profile reconstructed with the new algorithm. (b) FWM efficiency curve for the simulated fiber and FWM efficiency of the reconstructed fiber.

\section{Four-Wave Mixing Efficiency Curves with}

Measurement Noise and Imperfect Knowledge of the Fiber Parameters

We also wanted to test the robustness of this approach to errors in measurement of the fiber parameters (in our case, the fiber parameters are attenuation $\alpha$, length $L$, and dispersion slope $\left.\mathrm{d} D_{c} / \mathrm{d} \lambda\right)$. Therefore we tried to reconstruct the zero-dispersion wavelength profiles of the fibers by considering noisy fiber parameters. The tolerances of the parameters involved in the fitting depend only slightly on the measurement conditions. For example, in the attenuation tests, with smaller $\Delta \lambda s(1-3.5 \mathrm{~nm}$ for the fibers analyzed) the tolerance of the parameters to measurement errors can be very large (sometimes more than $30 \%$ ). For larger $\Delta \lambda s(4-6 \mathrm{~nm})$ the tolerance can be reduced to $\pm 5 \%$. For the length tests we obtained reasonable tolerances of more than $\pm 5 \%$ for all the reconstructions performed (for a 10-km fiber this means allowed errors of $\pm 500 \mathrm{~m})$. This means that simple optical time-domain reflectometer measurements should yield sufficiently precise results to be used as inputs into this reconstruction algorithm. We also ran all

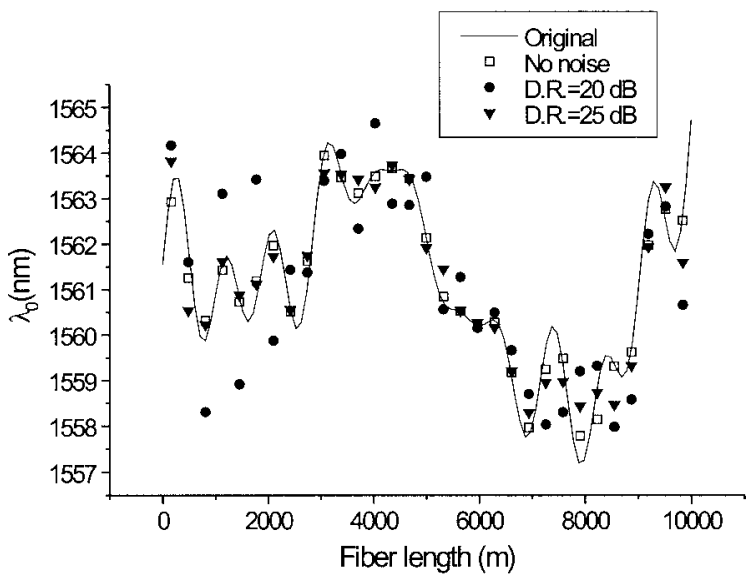

(a)

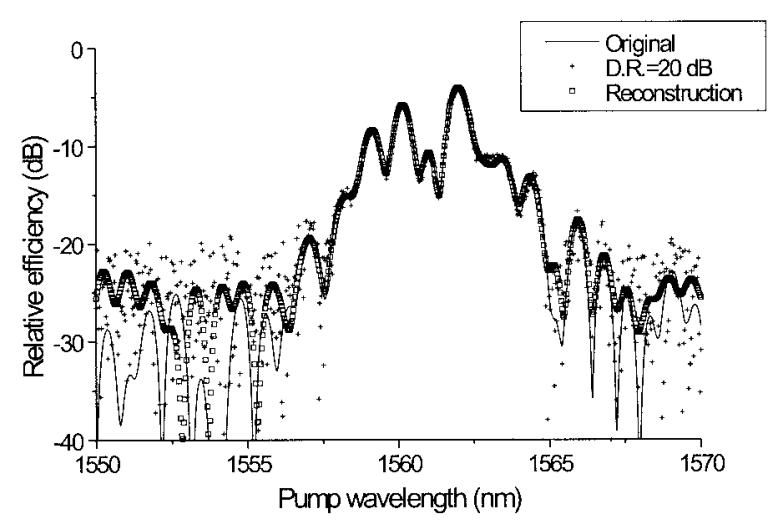

(b)

Fig. 5. (a) Zero-dispersion wavelength map of nonuniform fiber with $L_{c} \sim 1 \mathrm{~km}$ in the $1550-\mathrm{nm}$ window and profile reconstructed with the new algorithm for various levels of noise. (b) Original FWM efficiency curve of the simulated fiber with no noise; measured FWM efficiency including noise and reconstructed efficiency.

the tests with efficiencies acquired under dynamic range limitation conditions. The presence of noise did not reduce the tolerances significantly. A sample reconstruction that included noise in the fiber parameters (with the same DSF as we had used before) is shown in Fig. 6 . As can be seen, successful reconstructions were achieved both in the case of dynamic range limitation and in the case of noisefree efficiency.

\section{Limitations of This Technique Caused by Polarization-Mode Dispersion}

Until now we have considered a model of a fiber with negligible PMD. In this model the polarization states of the two interacting waves are aligned at the input of the fiber, and they keep this alignment through their propagation along the fiber. Thus, efficiency of the FWM process in each point is governed only by the phase mismatch between the two wavelengths involved in the process. However, it is well known that the FWM interaction is 


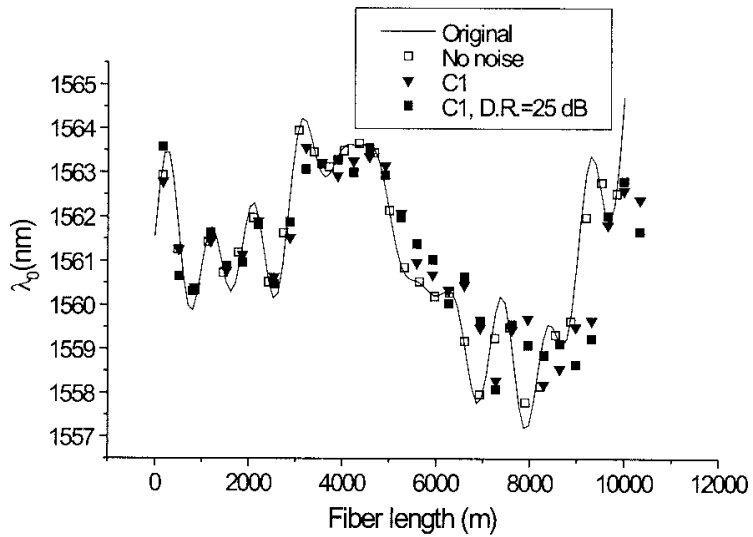

Fig. 6. Reconstruction under conditions of noise in the fiber parameters. The attenuation has an $11 \%$ underestimation, the length has a $5 \%$ overestimation, and the dispersion slope has a $10 \%$ overestimation (all these conditions are labeled as $\mathrm{C} 1$ in the figure). Results were obtained with noisy and nonnoisy FWM efficiency data.

polarization dependent. In fact, the efficiency of FWM is known to be proportional to $\mid \mathbf{E}_{1}\left(z, \lambda_{1}\right) \mathbf{E}_{2}(z$, $\left.\lambda_{2}\right)\left.\right|^{2}$. In the consideration of PMD the evolution of the polarization states along the fiber is stochastic, and a random modulation is introduced into the FWM efficiency.

In the strict sense, there is no systematic way to remove this modulation from the measured FWM efficiency, but we can avoid the effect of PMD if the wavelength separation $(\Delta \lambda)$ is sufficiently small, because smaller wavelength detunings will give a higher correlation between $\mathbf{s}_{1}$ and $\mathbf{s}_{2}$. Therefore, as the spatial resolution limit is given by $\Delta \lambda$, the PMD of the fiber will limit the maximum achievable spatial resolution. To gain further insight into the practical limit imposed on $\Delta \lambda$ by the PMD of the fiber we can use a first-order approximation:

$$
\Delta \xi(L, \Delta \omega) \simeq \Delta \tau(\omega) \Delta \omega
$$

where $\Delta \xi$ is the angle between the two states of polarization in the Poincaré sphere. To guarantee that $\Delta \xi$ remains reasonably close to zero we can set

$$
\Delta \omega \leq \frac{0.3}{\sqrt{\left\langle\Delta \tau^{2}\right\rangle}} \Rightarrow \Delta \lambda \leq \frac{0.3 \lambda^{2}}{2 \pi c \sqrt{\left\langle\Delta \tau^{2}\right\rangle}}
$$

Inasmuch as the autocorrelation of the PMD vector is known, ${ }^{14}$ we can determine the wavelength span in which the first-order approximation that we have used holds:

$$
\Delta \omega \leq \frac{0.6}{\sqrt{\left\langle\Delta \tau^{2}\right\rangle}} \Rightarrow \Delta \lambda \leq \frac{0.6 \lambda^{2}}{2 \pi c \sqrt{\left\langle\Delta \tau^{2}\right\rangle}} .
$$

Therefore the criterion expressed in relation (14) can be used.

\section{Conclusion}

The problem of reconstructing the zero-dispersion wavelength map of an optical fiber from its four-wave mixing efficiency has been linked to the classic problem of phase retrieval. We have proposed a new algorithm for solving this problem, which we believe is more efficient than any other error-minimization technique. Because of the special characteristics of this problem, the new algorithm is based on adding new restrictions to the basic error-reduction algorithm developed by Fienup. To our knowledge, this is one of the first times that this algorithm has proved useful for a one-dimensional problem. We have tested this algorithm on a variety of zero-dispersion wavelength profiles and in noisy conditions. In all cases the algorithm proved to be more robust than the error-minimization technique. The resolution of the mapping depends strongly on the measurement conditions, but practical resolutions of less than $100 \mathrm{~m}$ can be achieved with a simple experimental setup. The ultimate resolution of this technique is limited by the polarization mode dispersion of the fiber.

We acknowledge financial support from the Spanish Ministerio de Ciencia y Tecnología through project TIC2000-2003. We thank Jesús González of Telefónica de España for his interest. M. GonzálezHerráez acknowledges support from the Comunidad Autónoma de Madrid through a "Formación de Personal Investigador" grant.

\section{References}

1. S. Nishi and M. Saruwatari, "Technique for measuring the distributed zero dispersion wavelength of optical fibers using pulse amplification caused by modulation instability," Electron. Lett. 31, 225-226 (1995).

2. L. F. Mollenauer, P. V. Mamyshev, and M. J. Neubelt, "Method for facile and accurate measurement of optical fiber dispersion maps," Opt. Lett. 21, 1724-1726 (1996).

3. I. Brener, P. P. Mitra, D. D. Lee, D. J. Thomson, and D. L. Philen, "High-resolution zero-dispersion wavelength mapping in single-mode fiber," Opt. Lett. 23, 1520-1522 (1998).

4. M. Eiselt, R. M. Jopson, and R. H. Stolen, "Nondestructive position-resolved measurement of the zero-dispersion wavelength in an optical fiber," J. Lightwave Technol. 15, 135-143 (1997).

5. J. B. Schlager, "Zero-dispersion in optical fibers from cw fourwave mixing efficiency," in LEOS '98 (Institute of Electrical and Electronic Engineers, Orlando, Fla., 1998), Vol. 2, pp. 309-331.

6. M. L. Hernanz, M. Gonzalez-Herraez, and P. Corredera, "Zero-dispersion wavelength mapping in single-mode optical fibers using cw four-wave mixing," in 6th Optical Fibre Measurement Conference (OFMC '01) Proceedings (National Physical Laboratory, Teddington, Middlesex, UK, 2001), pp. 225-229.

7. M. L. Hernanz, P. Corredera, and M. Gonzalez-Herraez, "Selection of uniform fibers as standards for chromatic dispersion using cw four-wave mixing," in 6th Optical Fibre Measurement Conference (OFMC '01) Proceedings (National Physical Laboratory, Teddington, Middlesex, UK, 2001), pp. 235-239.

8. K. Inoue, "Four-wave mixing in an optical fiber in the zerodispersion wavelength region," J. Lightwave Technol. 10, 1553-1561 (1992). 
9. S. Song, C. T. Allen, K. R. Demarest, and R. Hui, "Intensitydependent phase-matching effects on four-wave mixing in optical fibers," J. Lightwave Technol. 17, 2285-2290 (1999).

10. H. Onaka, K. Otsuka, H. Miyata, and T. Chikama, "Measuring the longitudinal distribution of four-wave mixing efficiency in dispersion-shifted fibers," IEEE Photon. Technol. Lett. 6, 1454-1456 (1994).

11. M. Karlsson, "Four-wave mixing in fibers with randomly vary- ing zero-dispersion wavelength,” J. Opt. Soc. Am. B 15, 22692275 (1998).

12. J. R. Fienup, "Reconstruction of an object from the modulus of its Fourier transform," Opt. Lett. 3, 27-29 (1978).

13. M. Nieto-Vesperinas and J. A. Mendez, "Phase retrieval by Monte Carlo methods," Opt. Commun. 59, 249-254 (1986).

14. M. Karlsson and J. Brentel, "Autocorrelation function of the polarization-mode dispersion vector," Opt. Lett. 24, 939-941 (1999). 